\section{Associação do índice CPO-D com indicadores sócio-econômicos e de provisão de serviços odontológicos no Estado do Paraná, Brasil}

\author{
Association of the DMFT index \\ with socioeconomic and dental services \\ indicators in the state of Paraná, Brazil
}

Márcia Helena Baldani 1
Ana Glória Godoi Vasconcelos 2
José Leopoldo Ferreira Antunes ${ }^{3}$

Introdução

${ }^{1}$ Faculdade de Odontologia, Universidade Estadual de Ponta Grossa,

Ponta Grossa, Brasil.

2 Departamento

de Epidemiologia e Métodos Quantitativos em Saúde,

Escola Nacional de Saúde Pública, Fundação Oswaldo Cruz, Rio de Janeiro, Brasil.

3 Faculdade de Odontologia, Universidade de São Paulo,

São Paulo, Brasil.

Correspondência Márcia Helena Baldani, Faculdade de Odontologia, Universidade Estadual de Ponta Grossa. Av. Carlos Cavalcante 4748, Bloco M, Campus de Uvaranas, Ponta Grossa, PR 84030-000, Brasil. marcia@convoy.com.br

\begin{abstract}
This ecological study investigated the associations between dental caries, socioeconomic indicators, and the supply of dental services in the State of Paraná, Brazil, for the year 1996. Two types of information were collected: (1) data on caries prevalence (DMFT at 12 years) for the municipalities (or counties) in the State; (2) data on socioeconomic conditions and the supply of dental services. Based on simple linear regression analysis, the study demonstrated $a$ significant correlation between the caries index in municipalities and various social and dental services supply indicators. Results of the analysis of multiple linear regression showed that one indicator of income inequality remained significantly associated with dental caries, demonstrating that the worst oral health conditions cannot be dissociated from income disparities. A significant negative correlation was observed between DMFT and the proportion of the population that received fluoridated water, principally in the municipalities with the worst income inequality indicators. This highlights the importance of fluoridation for the reduction of caries rates, as well as to attenuate the impact of socioeconomic inequalities on the prevalence of dental caries.
\end{abstract}

Health Resources; Socioeconomic Factors; Fluoridation; DMF Index
A cárie dentária é uma doença que tem acompanhado a espécie humana ao longo da história. Junto com a industrialização, a prevalência desta aumentou até que praticamente toda a população foi afetada 1 . Porém, a partir das décadas de 60 e 70, uma redução contínua nos níveis de cárie tem sido observada nos países mais desenvolvidos. Existe um consenso de que um declínio acentuado e real está ocorrendo entre crianças nos países industrializados e em alguns países em desenvolvimento, inclusive no Brasil, tendo como possíveis causas: a utilização em larga escala de dentifrícios fluorados, a fluoração das águas de abastecimento público, as melhorias nas condições de vida das populações, alterações nos padrões dietéticos e mudanças nos critérios de diagnóstico da doença 2 .

Além desses fatores, alguns autores citam as melhorias nos serviços de saúde bucal como responsáveis pelo declínio da cárie 3,4,5. Porém, Peterson \& Bratthall 6 argumentam que o declínio da cárie ocorreu em países distantes uns dos outros e com diferentes sistemas de serviços de saúde bucal. Renson et al. 7 citam o exemplo do Japão, país desenvolvido que, apesar de apresentar o menor consumo de açúcar dentre os países industrializados e de possuir um serviço odontológico curativo e preventivo bem estruturado, somente passou a apresentar declínio 
na prevalência de cárie a partir da introdução em larga escala dos dentifrícios fluorados.

Nadanovsky \& Sheiham 8 afirmam que as mudanças sócio-econômicas (bem mais do que a contribuição dos serviços de saúde) têm um papel relevante na redução observada nos índices de cárie dentária. O estudo realizado com base em dados de dezoito países industrializados demonstrou que os serviços odontológicos, medidos pela proporção dentista/população, explicaram $3 \%$ da redução observada no CPO-D médio das crianças de 12 anos, durante os anos 70 e meados dos 80, enquanto que os fatores sociais explicaram $65 \%$ da redução observada. Observaram que a disponibilidade de serviços odontológicos não foi importante para explicar as diferenças nas variações do CPO-D aos 12 anos nos países estudados. Países com relações dentista/população e sistemas de atenção diferentes apresentaram valores de variação de CPO-D muito similares até a metade da década de 80 .

Nas últimas décadas, a condição social tem sido enfatizada como importante determinante da situação de saúde bucal 2, e estudos têm demonstrado que o declínio da cárie dentária vem sendo acompanhado pela polarização da doença nos grupos menos privilegiados 5. Vários trabalhos têm abordado a associação entre desenvolvimento social e cárie dentária, tanto no campo do indivíduo 9,10,11,12,13, como no ecológico 8,14,15,16. Estes estudos revelam que a prevalência de cárie dentária expressa pelo do Índice CPO-D, mostra-se significativamente pior para populações de baixo nível sócio-econômico.

A importância da fluoração das águas de abastecimento público para o controle da cárie dentária também tem sido largamente descrita na literatura 17,18, sendo que a Divisão de Saúde Bucal do CDC (Centers for Disease Control and Prevention) 19 afirma que este método foi o principal responsável pelo declínio na prevalência de cárie durante a segunda metade do século XX.

Com a difusão de outras formas de utilização do flúor, principalmente os dentifrícios fluorados, alguns autores têm sugerido que, em alguns países, os níveis de cárie não aumentam mesmo após a interrupção da fluoração das águas 20,21. Porém, estudos têm indicado que a implementação desta medida ainda se faz importante, principalmente em áreas de maior privação social 22,23,24. Segundo estes, a fluoração das águas tem um duplo papel: de redução nos níveis de cárie entre as crianças e, com isso, redução dos efeitos das desigualdades sócio-econômicas na experiência de cárie.
Associações significativas entre cárie dentária e indicadores sócio-econômicos nos municípios do Estado do Paraná para o ano de 1996 já foram previamente demonstradas por Baldani et al. 25. Diante disso, o presente estudo tem por objetivo contemplar a análise realizada por esses autores com a verificação da associação da oferta de serviços odontológicos, da fluoração das águas de abastecimento público e da condição social com a variação do Índice CPO-D.

\section{Métodos e técnicas}

Este é um estudo de natureza quantitativa, sendo classificado como ecológico e transversal. As variáveis estudadas foram o valor do Índice CPO-D aos 12 anos para 357 dos 371 municípios do Estado do Paraná em 1996, e alguns dos indicadores sócio-econômicos indicados na pesquisa anterior. Foram coletados dados adicionais relativos à oferta de serviços odontológicos públicos e privados, bem como a proporção de população coberta com água de abastecimento fluorada.

Os indicadores de prevalência de cárie foram obtidos junto à Secretaria de Estado da Saúde do Paraná (SES-PR), e correspondem ao resultado de um levantamento epidemiológico em saúde bucal conduzido sob responsabilidade técnico-científica desta, em 1996, com o objetivo de identificar o Índice CPO-D aos 12 anos, segundo metodologia da Organização Mundial da Saúde 26. O relatório desse levantamento afirma ter sido realizada a calibração dos examinadores, mas não fornece informações sobre indicadores de concordância intra e interexaminadores. Nesse sentido, o presente artigo admitiu a viabilidade da análise dos dados obtidos naquele estudo transversal. As informações relativas à oferta de água fluorada para os municípios do estado também foram disponibilizadas pela SES-PR, por meio de relatório oficial.

Os aspectos sociais e de oferta de serviços odontológicos foram evidenciados pelos diferentes indicadores obtidos junto aos bancos de dados do DATASUS (Departamento de Informática do SUS) 27 e do IBGE (Fundação Instituto Brasileiro de Geografia e Estatística) 28, a saber: (a) relativos à renda: renda familiar média "per capita" (razão entre número de salários mínimos e número de integrantes da família), Índice de Theil (indicador da desigualdade na distribuição de renda, descrito por Theil em 1971 29), e renda insuficiente (porcentagem de população vivendo com renda média familiar 
per capita inferior a meio salário mínimo); (b) relacionados à moradia: aglomeração domiciliar (porcentagem de população vivendo em domicílios com mais de dois moradores por dormitório) e proporção de domicílios ligados à rede de águas; (c) relativos à escolaridade: coeficiente de analfabetismo em adultos (15 ou mais anos de idade), anos médios de estudo e proporção de crianças (de 7 a 14 anos) que não freqüentavam escola; e (d) relativos à oferta de serviços: número de consultórios odontológicos no serviço público e número de cirurgiõesdentistas inscritos no Conselho Regional de Odontologia (CRO) por 1.000 habitantes.

A pesquisa constou de uma primeira etapa, exploratória, na qual os dados referentes ao índice CPO-D aos 12 anos e aos demais indicadores, para municípios do Estado do Paraná, foram analisados e interpretados segundo estatísticas descritivas.

Para verificar se as variáveis de estudo seguem o padrão da distribuição normal foram construídos gráficos (histogramas) de distribuição, bem como aplicou-se o teste de Kolmogorov-Smirnov para Normalidade 30 . As variáveis que não apresentaram distribuição normal passaram por transformação logarítmica, o que reduziu a assimetria da distribuição 31 .

No presente trabalho, pretendeu-se quantificar as associações de interesse por meio de modelos de regressão linear, desenvolvidos em duas etapas. Numa primeira etapa repetiu-se a análise realizada por Baldani et al. 25 e verificou-se a associação entre prevalência de cárie dentária e os diversos indicadores de desenvolvimento social, de oferta de flúor nas águas e de serviços odontológicos por meio de modelos de regressão linear simples, considerando o Índice CPO-D aos 12 anos como variável dependente.

Como resultado da primeira etapa foram identificadas as variáveis mais fortemente correlacionadas com a prevalência de cárie dentária nos diversos grupos de indicadores. Posteriormente, analisou-se a associação conjunta dos indicadores identificados na etapa anterior e o índice de cárie dentária por meio de regressão linear múltipla. O método utilizado para a seleção das variáveis foi o stepwise, considerando-se o nível de significância para a inclusão das variáveis de $5 \%$ e o de exclusão de $10 \%$. Para o modelo selecionado procedeu-se análise de resíduos com o objetivo de checar as suposições do modelo de regressão linear e identificação de outliers 31 . Para a análise dos dados foi utilizado o programa SPSS versão 8.0.

Para avaliar o papel da fluoração das águas de abastecimento público na redução da cárie dentária foram adotados dois procedimentos. O primeiro consistiu em dividir os municípios em quatro grupos de acordo com os quartis da variável proporção de população que recebe água fluorada. Em seguida, aplicou-se análise de variância a fim de verificar se os valores médios de CPO-D entre os grupos diferiam significativamente.

Um segundo procedimento foi utilizado para testar a hipótese de que quanto piores os indicadores de disparidade de renda, ou maior a desigualdade social, mais efetivo é o papel do flúor na redução dos índices de cárie 23,24. Esse procedimento consistiu em separar os municípios em dois grupos: um com menores e outro com maiores valores dos indicadores de desigualdade social, considerando-se, para tanto, a mediana dos indicadores renda insuficiente e Índice de Theil como ponto de corte. Essas variáveis foram selecionadas diante de evidências apontadas pela literatura de que são as sociedades menos igualitárias que apresentam maiores níveis de doenças 15. Procedeu-se então à análise de regressão múltipla para cada grupo, forçando a entrada das variáveis previamente selecionadas pelo procedimento stepwise para o conjunto global de municípios. Nessa etapa da análise incluiu-se no grupo de variáveis previamente selecionadas a variável população que recebe água fluorada como possível candidata ao modelo final no lugar da variável proporção de domicílios ligados à rede de água.

A variável população que recebe água fluorada não apresentou distribuição normal após a transformação log. Devido à importância desta variável na determinação da cárie dentária, amplamente demonstrada pela literatura, analisou-se sua possível contribuição para a redução da cárie dentária por meio do modelo de regressão múltipla, tendo-se o cuidado de interpretar seus resultados dentro das limitações impostas pela violação dessa suposição.

\section{Resultados}

De acordo com a análise descritiva prévia 25 , tem-se que a média de CPO-D para o Estado do Paraná é de 5,15 , sendo que pouco mais da metade dos municípios têm um valor abaixo dessa média. A Tabela 1 apresenta outros valores médios dos indicadores, sendo que alguns deles chamam a atenção: $56,16 \%$ da população vive com renda insuficiente; o coeficiente de analfabetismo é de $19,92 \%$; a proporção média de domicílios ligados à rede de águas é de $57,81 \%$, e da população que recebe água fluo- 
Estatísticas descritivas para as variáveis de cárie dentária, desenvolvimento social e provisão de serviços odontológicos dos municípios do Estado do Paraná, Brasil.

\begin{tabular}{|c|c|c|c|c|c|c|c|c|c|}
\hline Variável & $\mathbf{n}$ & Média & DP & CV & Mínimo & $P_{25}$ & Mediana & $P_{75}$ & Máximo \\
\hline CPO-D & 357 & 5,15 & 1,87 & 36,24 & 1,24 & 3,88 & 4,98 & 6,17 & 12,90 \\
\hline Renda familiar média per capita & 314 & 0,83 & 0,30 & 35,90 & 0,35 & 0,62 & 0,77 & 1,00 & 2,56 \\
\hline Índice de Theil & 314 & 0,53 & 0,13 & 24,01 & 0,22 & 0,45 & 0,51 & 0,61 & 1,01 \\
\hline Renda insuficiente & 314 & 56,16 & 13,73 & 24,45 & 13,48 & 47,43 & 57,50 & 67,10 & 87,42 \\
\hline Aglomeração domiciliar & 314 & 32,07 & 12,91 & 40,27 & 15,50 & 21,00 & 31,60 & 46,00 & 49,10 \\
\hline $\begin{array}{l}\text { Domicílios com ligação } \\
\text { à rede de água }\end{array}$ & 313 & 57,81 & 20,25 & 35,03 & 14,49 & 42,32 & 60,32 & 74,45 & 97,21 \\
\hline População que recebe água fluorada & 354 & 58,43 & 30,07 & 51,46 & 0,00 & 39,19 & 61,48 & 82,80 & 100,00 \\
\hline Coeficiente de analfabetismo & 314 & 19,92 & 6,29 & 31,60 & 5,30 & 15,20 & 20,10 & 24,40 & 39,90 \\
\hline Anos médios de estudo & 314 & 3,57 & 0,75 & 21,10 & 1,80 & 3,10 & 3,50 & 4,00 & 7,40 \\
\hline Crianças (7 a 14 anos) sem escola & 314 & 19,75 & 7,32 & 37,07 & 3,40 & 14,50 & 18,75 & 23,20 & 58,60 \\
\hline $\begin{array}{l}\text { Número de consultórios/ } \\
1.000 \text { habitantes/SUS }\end{array}$ & 321 & 0,33 & 0,50 & 151,21 & 0,00 & 0,16 & 0,27 & 0,43 & 0,68 \\
\hline $\begin{array}{l}\text { Número total de dentistas/ } \\
1.000 \text { habitantes }\end{array}$ & 294 & 0,49 & 0,37 & 75,10 & 0,04 & 0,23 & 0,38 & 0,61 & 2,56 \\
\hline
\end{tabular}

$C V=$ Coeficiente de variação; $P_{25}=$ Porcentil $25 ; P_{75}=$ Porcentil 75.

rada é de 58,43\%. Com relação ao número de cirurgiões-dentistas por habitante observa-se uma proporção média de 4,9/10 mil habitantes. Observou-se elevada variabilidade nos indicadores de oferta de serviços odontológicos.

A Tabela 2 mostra os coeficientes de correlação de Pearson para o Índice CPO-D e as diversas variáveis estudadas. Verifica-se que todas as variáveis apresentam correlação significativa em nível de 5\% com o Índice CPO-D. A prevalência de cárie e o indicador social renda insuficiente apresentaram correlação positiva, indicando a tendência de municípios com piores condições de vida apresentarem maiores índices do agravo. Quanto à variável total de cirurgiões-dentistas observou-se correlação negativa, o que pode indicar uma maior concentração destes nos municípios com menores índices de cárie.

Os indicadores que apresentaram maior correlação com o Índice CPO-D nos grupos de renda, habitação, escolaridade e oferta de serviços odontológicos pré-selecionados para a análise de regressão múltipla encontram-se destacados na Tabela 2. Do grupo relativo à renda foi selecionada a variável renda insuficiente; do grupo de habitação selecionou-se a variável: domicílios ligados à rede de águas; daquele grupo relacionado à educação a variável selecionada foi: anos médios de estudo; e do relativo à oferta de serviços foi escolhida a variável: número de dentistas inscritos no CRO por 1.000 habitantes.
Na Tabela 3 pode-se observar o modelo de regressão múltipla obtido pelo método stepwi$s e$, bem como a ordem de entrada das variáveis e a respectiva contribuição em termos de porcentual da variação explicada, além das estimativas dos parâmetros por mínimos quadrados ordinários. O modelo final obtido inclui renda insuficiente e número total de cirurgiões-dentistas, sendo a renda insuficiente a primeira variável a entrar. A variável renda insuficiente foi estatisticamente significativa no modelo descrito ( $\mathrm{p}<0,001$ ), bem como a variável número de cirurgiões-dentistas inscritos no CRO por 1.000 habitantes ( $p=0,047)$. Porém, ao se comparar a ordem de entrada das variáveis no modelo, verifica-se a reduzida contribuição parcial daquela de serviço odontológico na explicação das variações no índice CPO-D.

Complementando essa etapa do estudo, realizou-se a análise de resíduos para o modelo final obtido, na qual pôde-se identificar a presença de outliers. Para verificar a influência destes nos resultados da regressão, os municípios correspondentes a esses resíduos foram excluídos (doze) e ajustou-se o modelo final, apenas com as variáveis número total de dentistas e renda insuficiente, aos municípios restantes $(\mathrm{n}=266)$.

Observou-se que, após a remoção dos outliers, o modelo final obtido apontou apenas uma única variável significativa, a renda insuficiente $(p<0,001)$, que sozinha explica $13,1 \%$ da variação na média de CPO-D para os mu- 
nicípios do Estado do Paraná. Como informação adicional, verificou-se que o coeficiente de determinação ajustado passou de 0,125 para 0,131 .

Na Tabela 4 podem ser verificados os resultados da análise de regressão múltipla para o CPO-D quando, no grupo de variáveis previamente selecionadas, substitui-se a variável proporção de domicílios ligados à rede de água por proporção de população que recebe água fluorada. O modelo final seleciona as variáveis proporção de população que recebe água fluorada e renda insuficiente, sendo excluída a variável total de dentistas inscritos no CRO. Verifica-se que o coeficiente da variável de acesso ao flúor foi estatisticamente significante no modelo descrito $(p=0,022)$, bem como a renda insuficiente $(p<0,001)$. Neste caso, o modelo multivariado obtido indica que a renda insuficiente e a proporção da população que recebe água fluorada explicam juntas $12,7 \%$ da variação do CPO-D no Estado do Paraná.

A Tabela 5 apresenta os modelos de regressão obtidos para dois grupos de municípios: um com menores e outro com maiores valores dos indicadores de desigualdade social, identificados com base nas variáveis: renda insuficiente e Índice de Theil. As variáveis que entraram na análise de regressão múltipla foram as selecionadas previamente pelo procedimento stepwise: renda insuficiente e proporção de população que recebe água fluorada. Verificase que para os municípios com menor desigualdade social (menor proporção de população com renda insuficiente e menor Índice de Theil), a variável significativamente associada com o Índice CPO-D foi renda insuficiente, não importando o indicador utilizado para a classificação do grupo. Na mesma tabela pode-se observar que, para os municípios com mais desigualdade social, a variável população que recebe água fluorada é significativamente associada com o CPO-D tanto para o grupo selecionado com base na mediana da renda insuficiente, como para o selecionado baseandose na mediana do Índice de Theil. Para este último, o coeficiente de determinação ajustado é de 0,156 e o modelo obtido inclui as variáveis renda insuficiente e população que recebe água fluorada.

\begin{tabular}{|c|c|c|}
\hline \multicolumn{3}{|c|}{$\begin{array}{l}\text { Análise de correlação entre o Índice CPO-D e as variáveis de desenvolvimento } \\
\text { social e de oferta de serviços odontológicos. }\end{array}$} \\
\hline Indicadores & $\begin{array}{l}\text { Coeficiente de } \\
\text { Correlação de Pearson }\end{array}$ & $\begin{array}{l}\text { Nível de } \\
\text { significância }\end{array}$ \\
\hline Renda familiar média per capita* & $-0,26$ & $p<0,001$ \\
\hline Índice de Theil* & 0,12 & $p=0,032$ \\
\hline Renda insuficiente & 0,31 & $p<0,001$ \\
\hline Aglomeração domiciliar* & 0,16 & $p=0,001$ \\
\hline Domicílios com ligação à rede de água & $-0,30$ & $p<0,001$ \\
\hline População que recebe água fluorada & $-0,30$ & $p<0,001$ \\
\hline Coeficiente de analfabetismo & 0,15 & $p=0,007$ \\
\hline Anos médios de estudo* & $-0,28$ & $p<0,001$ \\
\hline Crianças (7 a 14 anos) sem escola & 0,17 & $p=0,003$ \\
\hline $\begin{array}{l}\text { Número de consultórios SUS/ } \\
1.000 \text { habitantes*}^{*}\end{array}$ & 0,17 & $p=0,002$ \\
\hline $\begin{array}{l}\text { Número total de dentistas/ } \\
1.000 \text { habitantes }\end{array}$ & $-0,27$ & $p<0,001$ \\
\hline
\end{tabular}

* Logaritmo da variável.

Resultados do modelo de regressão linear múltipla (stepwise) para o Índice CPO-D.

\begin{tabular}{|c|c|c|c|c|c|}
\hline $\begin{array}{l}\text { Modelo por ordem } \\
\text { de entrada das variáveis }\end{array}$ & Estimativa & \multicolumn{3}{|c|}{ Coeficiente } & \\
\hline Constante & 2,508 & 0,414 & $P<0,001$ & 1,694 & 3,323 \\
\hline Renda insuficiente & 0,044 & 0,007 & $P<0,001$ & 0,030 & 0,058 \\
\hline \multicolumn{6}{|l|}{$2 R^{2}$ ajustado $=0,125$} \\
\hline Constante & 2,684 & 0,421 & $p<0,001$ & 1,856 & 3,513 \\
\hline Renda insuficiente & 0,035 & 0,008 & $p<0,001$ & 0,018 & 0,052 \\
\hline $\begin{array}{l}\text { Número total de dentistas/ } \\
1.000 \text { habitantes* }\end{array}$ & $-0,774$ & 0,387 & $p=0,047$ & $-1,536$ & $-0,013$ \\
\hline
\end{tabular}

* Logaritmo da variável. 
Resultados do modelo de regressão linear múltipla para o Índice CPO-D, após inclusão da variável de fluoração das águas de abastecimento público.

\begin{tabular}{|c|c|c|c|c|c|}
\hline $\begin{array}{l}\text { Modelo por ordem } \\
\text { de entrada das variáveis }\end{array}$ & \multicolumn{5}{|c|}{ Coeficiente } \\
\hline \multicolumn{6}{|l|}{$1 \mathrm{R}^{2}$ ajustado $=0,114$} \\
\hline Constante & 2,513 & 0,414 & $P<0,001$ & 1,698 & 3,329 \\
\hline Renda insuficiente & 0,044 & 0,007 & $P<0,001$ & 0,029 & 0,058 \\
\hline \multicolumn{6}{|l|}{$2 \mathrm{R}^{2}$ ajustado $=0,127$} \\
\hline Constante & 3,567 & 0,615 & $p<0,001$ & 2,356 & 4,778 \\
\hline Renda insuficiente & 0,035 & 0,008 & $p<0,001$ & 0,020 & 0,051 \\
\hline População que recebe água fluorada & $-0,009$ & 0,004 & $p=0,022$ & $-0,018$ & $-0,001$ \\
\hline
\end{tabular}

Tabela 5

Resultados do modelo de regressão linear múltipla para o Índice CPO-D,

segundo grupo de municípios apresentando menos e mais desigualdade social.

\begin{tabular}{|c|c|c|c|}
\hline \multirow[t]{2}{*}{ Indicador } & \multirow[t]{2}{*}{ Modelo } & \multicolumn{2}{|c|}{ Coeficiente } \\
\hline & & Estimativa & p-valor \\
\hline \multicolumn{4}{|c|}{$\begin{array}{l}\text { Municípios com menos } \\
\text { desigualdade social }\end{array}$} \\
\hline & R2 ajustado $=0,096$ & & \\
\hline \multirow[t]{4}{*}{ Renda insuficiente } & Constante & 2,601 & $p=0,001$ \\
\hline & Renda insuficiente & 0,052 & $p<0,001$ \\
\hline & População que recebe água fluorada & $-0,004$ & $p=0,318$ \\
\hline & $\mathrm{R} 2$ ajustado $=0,064$ & & \\
\hline \multirow[t]{3}{*}{ Índice de Theil } & Constante & 3,441 & $p<0,001$ \\
\hline & Renda insuficiente & 0,031 & $p=0,006$ \\
\hline & População que recebe água fluorada & $-0,003$ & $p=0,494$ \\
\hline \multicolumn{4}{|l|}{$\begin{array}{l}\text { Municípios com mais } \\
\text { desigualdade social }\end{array}$} \\
\hline & $\mathrm{R} 2$ ajustado $=0,047$ & & \\
\hline \multirow[t]{4}{*}{ Renda insuficiente } & Constante & 4,721 & $p=0,008$ \\
\hline & Renda insuficiente & 0,023 & $p=0,343$ \\
\hline & População que recebe água fluorada & $-0,016$ & $p=0,013$ \\
\hline & R2 ajustado $=0,156$ & & \\
\hline \multirow[t]{3}{*}{ Índice de Theil } & Constante & 4,517 & $p<0,001$ \\
\hline & Renda insuficiente & 0,030 & $p=0,007$ \\
\hline & População que recebe água fluorada & $-0,019$ & $p=0,002$ \\
\hline
\end{tabular}




\section{Discussão}

Valendo-se deste estudo buscou-se utilizar os recursos da epidemiologia para proporcionar uma noção sobre a situação da cárie dentária no Estado do Paraná, em 1996, bem como suas relações com condições sócio-econômicas e oferta de serviços odontológicos, e especificamente, com a fluoração das águas de abastecimento público. Os dados aqui relatados podem mostrar-se úteis para a definição de programas de prevenção e tratamento da doença, para o planejamento dos serviços ou para guiar políticas de saúde bucal no Estado do Paraná.

Apesar dos desenhos específicos e dos diferentes padrões de medidas e de amostra, os estudos realizados a partir da década de 80 têm demonstrado uma relação consistente entre condição sócio-econômica e cárie dentária em crianças 10,11,14,15. No Paraná, as correlações entre cárie dentária e os diversos indicadores de desenvolvimento social estudados mostraram-se significativas apesar dos baixos valores obtidos. Baixos valores de correlação, porém significativos, parece ser uma característica de estudos ecológicos com dados secundários que utilizam um grande número de unidades amostrais no Brasil. Valores semelhantes aos obtidos no presente estudo foram identificados por Santos \& Noronha 32 ao analisar as correlações entre indicadores sociais e mortalidade geral, e por causas em 144 bairros do Município do Rio de Janeiro. Esses autores encontraram coeficientes de correlação significativos variando de $-0,20$ a -0,52. A variável de renda foi a que apresentou os maiores valores de correlação dentre as variáveis sócio-econômicas utilizadas por eles. Leal \& Swarcwald 33, ao analisarem os coeficientes de correlação entre um indicador sócio-econômico composto e as taxas de mortalidade infantil para os 91 municípios do Estado do Rio de Janeiro, encontraram o valor $r=0,257$ para a idade de zero a 364 dias de vida, no período de 1979-1981 e r = 0,123 para 1990-1992.

No entanto, deve-se lembrar que a análise de dados agregados ao nível municipal não leva em consideração o impacto das desigualdades sociais em nível intramunicipal nas associações entre características populacionais. Além disso, conclusões baseadas em análise de dados agregados contemplam sempre o risco de sua validade ser afetada pela falácia ecológica. A despeito dessas considerações, a análise de dados espaciais viabiliza o estudo de associações entre níveis de agravo e seus possíveis fatores causais, em diversas situações nas quais não é possível a obtenção de informações pessoais e, desse modo, pode instruir os serviços de saúde na formulação de programas socialmente adequados e no direcionamento de recursos para as áreas mais carentes 34 .

A análise de regressão linear múltipla incluindo as variáveis pré-selecionadas (Tabela 2) identificou, após a análise de resíduos, apenas uma única variável como estatisticamente significante: a renda insuficiente. Resultado semelhante foi obtido por Patussi et al. 15 que demonstraram que o coeficiente Gini sozinho explicou $31 \%$ nos valores de CPO-D. No presente estudo, o modelo final explicou apenas $13,1 \%$ da variação do CPO-D. Neste caso, há que se considerar a elevada heterogeneidade dos municípios incluídos na análise, bem como uma possível variabilidade interna de cada município, já demonstrada por Camargo \& Moysés 35 e por Antunes et al. 16. Também não podem ser descartadas as considerações quanto à qualidade dos dados de CPO-D e dos demais dados secundários utilizados.

Ao se comparar os resultados obtidos com base nos métodos de análises utilizados nesse estudo para quantificar as associações, podese afirmar que a análise multivariada, por meio de regressão linear múltipla, não se mostrou superior em qualidade de ajuste à análise de associações no plano univariado, por meio de regressão linear simples. Uma explicação para isso seria o fato de as variáveis independentes serem parcialmente colineares e até certo ponto medirem características análogas das mesmas dimensões. Dessa forma, o modelo stepwise não autoriza a inclusão dessas múltiplas dimensões, selecionando sempre a variável de renda insuficiente como sendo a que melhor explica as variações no índice de CPO-D nos municípios analisados, excluindo as demais.

A renda familiar, bem como a inserção social, apresentaram relação direta na prevalência da cárie dentária conforme também foi demonstrado por Furlani 9, Nadanovsky 2, Peres et al. 12 e Baldani et al. 25. Na opinião de Marcenes \& Bönecker 36 no Brasil, como nos países desenvolvidos, a desigualdade social, em vez de privação material e social é o maior determinante da experiência de cárie medida pelo CPO-D. Quanto a esse aspecto, o estudo de Patussi 15 propiciou uma importante evidência científica para a teoria de que sociedades igualitárias apresentam melhores níveis de saúde do que as desiguais e, conseqüentemente, quanto mais desigual for a distribuição de renda na sociedade, maiores serão os contrastes em relação à saúde.

Gillcrist et al. 13 cogitaram que o acesso aos serviços odontológicos também é diferenciado para os diferentes grupos sociais. Crianças 
de famílias de baixo nível socioeconômico têm menor possibilidade de acesso aos serviços do que as de classes sociais mais altas, e conseqüentemente, também aos benefícios advindos destes como selantes, aplicações tópicas de flúor e uso de dentifrícios fluorados. Por outro lado, Nadanovsky \& Sheiham 8 demonstraram que as mudanças sócio-econômicas têm papel mais relevante na redução de cárie dentária do que a oferta de serviços odontológicos.

O presente estudo aponta uma correlação negativa entre cárie dentária e o número total de dentistas inscritos no CRO. A interpretação mais evidente seria a de que o maior número de cirurgiões-dentistas em um município contribui diretamente para um menor Índice CPOD. Porém, concordando com o que foi demonstrado por Nadanovsky \& Sheiham 8 , essa associação, medida pela análise de regressão linear simples, não se mostrou significativa quando controlada por indicadores sócio-econômicos e pela água fluorada, o que faz supor que a variável de serviços seja um possível fator de confusão.

Este estudo demonstrou também haver uma correlação positiva entre o Índice CPO-D e o número de consultórios disponíveis no serviço público (Tabela 2). Este resultado reflete uma possível tendência de que os municípios com piores indicadores sociais, mais pobres, portanto, sejam os que estejam oferecendo uma maior cobertura de serviços públicos à sua população. Para demonstrar essa hipótese, pesquisas específicas deveriam ser conduzidas.

Observou-se, neste estudo, correlação negativa entre o CPO-D e a proporção da população que recebe água fluorada (Tabela 2), o que pode ser interpretado como um indício de êxito dessa estratégia. O papel da fluoração das águas de abastecimento público na redução do CPO-D também tem sido largamente descrito na literatura 17,18,37. A Tabela 2 mostra que existe uma tendência de os municípios com maior proporção da população recebendo água fluorada apresentarem um menor CPO-D, o que foi confirmado pela análise de variância $(p<0,001)$ entre as médias de CPO-D para os quartis dessa variável.

Os resultados da análise de regressão linear verificados neste estudo para a variável proporção de população que recebe água fluorada (Tabela 4), devem ser vistos com cautela. Uma vez que a variável não apresentou distribuição normal, nem mostrou-se linearmente relacionada com a variável dependente do modelo, deixou de cumprir com dois dos pressupostos da análise de regressão linear. Portanto, os valores dos coeficientes obtidos podem es- tar sub ou superestimados. Porém, os resultados, apesar de não poderem ser indicados com segurança para estimativas de variações médias no Índice CPO-D, são significativos e estão de acordo com o que tem sido relatado na literatura, uma vez que sugerem a influência da presença do flúor nas águas de abastecimento público na variação da prevalência de cárie na população.

Informações complementares sobre o papel da fluoração das águas de abastecimento público na redução da cárie dentária mostram que essa influi significativamente e em maior intensidade na variação do CPO-D naqueles municípios com piores indicadores de desigualdade social (Tabela 5). O mesmo resultado não é obtido ao se substituir a variável proporção de população com acesso à água fluorada pela variável proporção de domicílios ligados à rede de água. Este achado concorda com os relatos de Slade et al. 22 e de Jones et al. 23 que concluíram que as regiões com piores condições sócio-econômicas são as que mais se beneficiam com a fluoração das águas, porque, além da redução nos níveis de cárie, esta medida contribui na redução das desigualdades sócio-econômicas na experiência de cárie. Resultados semelhantes também foram relatados por outros autores 9,24, os quais atribuem à fluoração das águas de abastecimento público a capacidade de reduzir sensivelmente o impacto negativo das desigualdades sócio-econômicas sobre a prevalência de cárie dentária.

Os resultados relatados no presente estudo indicam a importância da fluoração das águas de abastecimento público, principalmente naqueles municípios onde as desigualdades sociais são mais evidentes, concordando com o comentário de Narvai 38 segundo o qual o benefício é proporcionalmente maior justamente nos segmentos que não têm ou têm muito pouco acesso a outros fatores de proteção. Apesar das evidências do importante papel de outras medidas, como a fluoração dos dentifrícios 7,3,1,2, que foi instituída no Brasil na década de 80 , ao se levar em conta que a proporção média da população vivendo com renda menor do que meio salário mínimo/mês nos municípios do Estado do Paraná é de 56,16\% (Tabela 1), e que este estado é um dos melhores situados no Brasil do ponto de vista econômico, os resultados aqui obtidos permitem reforçar a opinião de Narvai 38 (p. 386), ao afirmar que a resistência em se implementar a fluoração das águas de abastecimento nos municípios ou de interromper o processo não são justificáveis sendo "juridicamente ilegais, cientificamente insustentáveis e socialmente injustas”. 


\section{Considerações finais}

Os resultados relatados neste trabalho evidenciam correlações significativas entre os indicadores de desenvolvimento social relacionados à renda, moradia e escolaridade com cárie dentária. Dentre estes, destaca-se um indicador de renda (renda insuficiente), demonstrando que as piores condições de saúde bucal não podem ser dissociadas das disparidades de renda. $\mathrm{O}$ acesso da população à água de abastecimento público fluorada também se afirmou como um fator importante na variação dos valores do Índice CPO-D no estado. Como indicação suplementar, foi evidenciada correlação entre cárie dentária e a oferta de serviços odontológicos medida pelo número de dentistas inscritos no CRO. Porém, a mesma não se mostrou significativa quando controlada pelos indicadores sócio-econômicos e pela água fluorada. Há que se considerar que as melhorias nas condições de saúde das populações têm sido marcadas

\section{Resumo}

Este estudo ecológico investigou as associações entre cárie dentária, indicadores sócio-econômicos e de oferta de serviços odontológicos no Estado do Paraná, Brasil, para o ano de 1996. Dois tipos de informações foram reunidos: (1) dados sobre prevalência de cárie dentária (CPO-D aos 12 anos) para os municípios do Estado; (2) dados relativos às condições sócio-econômicas e de oferta de serviços odontológicos. Com base em análise de regressão linear simples demonstrou-se correlação significativa entre o indice de cárie dentária nos municípios e os vários indicadores sociais e de oferta de serviços. Resultados da análise de regressão linear múltipla apontaram que apenas um indicador de desigualdade de renda permaneceu significativamente associado com cárie dentária, demonstrando que as piores condições de saúde bucal não podem ser dissociadas das disparidades de renda. Observou-se correlação negativa significativa entre o CPO-D e a proporção de população que recebe água fluorada, principalmente nos municípios com piores indicadores de desigualdade de renda. Nesse sentido, sublinha-se a importância desse benefício não só como recurso para a redução dos níveis de cárie, como também para atenuar o impacto das desigualdades sócio-econômicas sobre a prevalência de cárie dentária.

Recursos em Saúde; Fatores Sócio-econômicos; Fluoração; Índice CPO por intervenções no âmbito do coletivo, por políticas de Estado que têm privilegiado o investimento na melhoria da infra-estrutura urbana e serviços de atenção primária e de baixo custo. Uma reflexão mais aprofundada sobre o presente tema, indica que a possibilidade de intervenção sobre a cárie dentária deve ser direcionada, além da melhoria das condições de vida, para a esfera dos serviços de saúde, cujo uso adequado vai além da possibilidade de acesso da população. Há necessidade, também, de se implementar medidas e políticas compensatórias para amenizar os efeitos danosos das desigualdades sociais, uma vez que estas se tornam mais graves se estratégias preventivas não são capazes de prover ao menos benefícios equivalentes para grupos de baixo nível sócio-econômico. Este estudo corrobora o que tem sido descrito na literatura: a fluoração das águas de abastecimento público é uma estratégia capaz de reduzir as desigualdades em saúde bucal para os diferentes grupos da população.

\section{Colaboradores}

Este artigo é o resultado da dissertação de mestrado de M. H. Baldani, defendida na Escola Nacional de Saúde Pública, Fundação Oswaldo Cruz, em julho de 2002, sob a orientação de A. G. G. Vasconcelos e coorientação de J. L. F. Antunes. Todos os autores realizaram em conjunto todas as etapas do trabalho, desde a organização do banco de dados até as análises estatísticas. A. G. G. Vasconcelos e J. L. F. Antunes revisaram o texto da dissertação e do artigo.

\section{Referências}

1. Bratthall D, Hänsel-Peterson G, Sundberg H. Reasons for the caries decline: what do experts believe? Eur J Oral Sci 1996; 104:416-22.

2. Nadanovsky P. O declínio da cárie. In: Pinto VG, organizador. Saúde bucal coletiva. 4a Ed. São Paulo: Santos; 2000. p. 341-51.

3. Marthaler TM, O'Mullane DM, Vrbic V. The prevalence of dental caries in Europe 1990-1995. Caries Res 1996; 30:237-55.

4. Peres MAA, Rosa AGF. As causas da queda da cárie. RGO 1995; 43:160-4.

5. Weyne SC. A construção do paradigma de promoção de saúde: um desafio para as novas gerações. In: Kriger L, organizador. Promoção de saúde bucal. São Paulo: Artes Médicas; 1997. p. 1-26. 
6. Peterson HG, Bratthall D. The caries decline: a review of reviews. Eur J Oral Sci 1996; 104:436-43.

7. Renson CE, Crielaers PJA, Ibikunle SAJ, Pinto VG, Ross CB, Infirri JS, et al. Changing patterns of oral health and implications for health manpower: part I. Int Dent J 1985; 35:235-51.

8. Nadanovsky P, Sheiham A. Relative contribution of dental services to the changes in caries levels of 12-year-old children in 18 industrialized countries in the 1970s and early 1980s. Community Dent Oral Epidemiol 1995; 23:331-9.

9. Furlani PA. Influências dos aspectos sociais e culturais na prevalência da cárie dentária em escolares de Jaraguá do Sul [Monografia]. Curitiba: Universidade Federal do Paraná; 1993.

10. Moura MS, Pinto LAMS, Giro EMA, Cordeiro RCL. Cárie dentária relacionada ao nível socioeconômico em escolares de Araraquara. Revista Odontol UNESP 1996; 85:97-107.

11. Irigoyen ME, Maupome G, Mejia AM. Caries experience and treatment needs in a 6-to 12-yearold urban population in relation to socio-economic status. Community Dent Health 1999; 16: 245-9.

12. Peres KGA, Bastos JRM, Latorre MRDO. Severidade de cárie em crianças e relação com aspectos sociais e comportamentais. Rev Saúde Pública 2000; 34:402-8.

13. Gillcrist JA, Brumley DE, Blackford JU. Community socioeconomic status and children's dental health. J Am Dent Assoc 2001; 132:216-22.

14. Lalloo R, Myburgh NG, Hobdell MH. Dental caries, socio-economic development and national oral health policies. Int Dent J 1999; 49:196202.

15. Patussi MP, Marcenes W, Croucher R, Sheiham A. Social deprivation, income inequality, social cohesion and dental caries in Brazilian school children. Soc Sci Med 2001; 53:915-25.

16. Antunes JLF, Frazão P, Narvai PC, Bispo CM, Pegoretti $\mathrm{T}$. Spatial analysis to identify differentials in dental needs by area-based measures. Community Dent Oral Epidemiol 2002; 30:133-42.

17. Basting RT, Pereira AC, Meneghim MC. Avaliação da prevalência de cárie dentária em escolares do Município de Piracicaba, SP, Brasil, após 12 anos de fluoretação das águas de abastecimento público. Rev Odontol Univ de São Paulo 1997; 11:28792.

18. De Liefde B. The decline of caries in New Zealand over the past 40 years. N Z Dent J 1998; 94:109-13.

19. Centers for Disease Control and Prevention. Achievements in Public Health, 1900-1999: fluoridation of drinking water to prevent dental caries. JAMA 2000; 283:1283.

20. Seppä L, Kärkkäinen S, Hausen H. Caries frequency in permanent teeth before and after discontinuation of water fluoridation in Kuopio, Finland. Community Dent Oral Epidemiol 1998; 26:256-62.

21. Kunzel W, Fischer T. Caries prevalence after cessation of water fluoridation in La Salud, Cuba. Caries Res 2000; 34:20-5.

22. Slade GD, Spencer AJ, Davies MJ, Stewart JF. Influence of exposure to fluoridated water on socioeconomic inequalities in children's caries ex- perience. Community Dent Oral Epidemiol 1996; 24:89-100.

23. Jones CM, Taylor GO, Whittle JG, Evans D, Trotter DP. Water fluoridation, tooth decay in 5 year olds, and social deprivation measured by the Jarman score: analysis of data from British dental surveys. Br Med J 1997; 315:514-8.

24. Jones CM, Worthington H. Water fluoridation, poverty and tooth decay in 12-year-old children. J Dent 2000; 28:389-93.

25. Baldani MH, Narvai PC, Antunes JLF. Cárie dentária e condições socioeconômicas no Estado do Paraná, Brasil, 1996. Cad Saúde Pública 2002; 18:755-63.

26. Organização Mundial da Saúde. Levantamentos básicos em saúde bucal. 3ạ Ed. São Paulo: Editora Santos; 1991.

27. Departamento de Informática do SUS. Informações de saúde http://www.datasus.gov.br/ (acessado em 26/Mar/2001).

28. Fundação Instituto Brasileiro de Geografia e Estatística. Sistema IBGE de Recuperação Automática - SIDRA http://www.sidra.ibge.gov.br/ (acessado em 26/Mar/2001).

29. Theil H. Principles of econometrics. New York: Wiley; 1971.

30. Daniel WW. Biostatistics: a foundation for analysis in the health sciences. 6 th Ed. New York: Wiley; 1995.

31. Kleinbaum DG, Kupper LL, Muller KE, Nizan A. Applied regression analysis and multivariable methods. 3rd Ed. Belmont: Duxbury Press; 1998.

32. Santos MS, Noronha CP. Padrões espaciais de mortalidade e diferenciais sócio-econômicos na cidade do Rio de Janeiro. Cad Saúde Pública 2001; 17:1099-110.

33. Leal MC, Szwarcwald CL. Características de mortalidade neonatal no Estado do Rio de Janeiro na década de 80: uma visão espaço - temporal. Rev Saúde Pública 1997; 31:457-65.

34. Schwartz S. The fallacy of the ecological fallacy: the potential misuse of a concept and the consequences. Am J Public Health 1994; 84:819-24.

35. Camargo AL, Moysés SJ. A saúde bucal no Município de Curitiba. Divulg Saúde Debate 1996; 13: 54-65.

36. Marcenes W, Bonecker MJS. Aspectos epidemiológicos e sociais das doenças bucais. In: Buischi YP, organizador. Promoção de saúde bucal na clínica odontológica. São Paulo: Artes Médicas; 2000. p. 75-98.

37. Azcurra AI, Battellino LJ, Calamari SE, Cattoni STD, Kremer M, Lamberghini FC. Estado de salud bucodental de escolares residentes en localidades abastecidas com agua de consumo humano de muy alto y muy bajo contenido de fluoruros. Rev Saúde Pública 1995; 29:364-75.

38. Narvai PC. Cárie dentária e flúor: uma relação do século XX. Ciênc Saúde Coletiva 2000; 5:381-92.

Recebido em 28/Fev/2003

Versão final reapresentada em 11/Jun/2003

Aprovado em 01/Out/2003 